\title{
Patient, Provider and System Factors Impacting on the Diagnosis and Management of Lung Cancer Care in Australia
}

\author{
Moyez Jiwa ${ }^{1}$, Patricia M. Davidson², Phillip J. Newton ${ }^{2}$, Michelle L. DiGiacomo, ${ }^{1,2}$, Sarah J. McGrath ${ }^{2}$, \\ Cornelius J. Lotriet ${ }^{1}$
}

${ }^{1}$ Curtin Health Innovation Research Institute, Curtin University, Perth, Australia; ${ }^{2}$ Centre for Cardiovascular and Chronic Care, Faculty of Health, University of Technology Sydney, Sydney, Australia.

Email:m.jiwa@curtin.edu.au

Received April 30 ${ }^{\text {th }}$, 2012; revised May 31 $1^{\text {st }}$ 2012; accepted June $20^{\text {th }}, 2012$

\begin{abstract}
Background: Lung cancer is the leading cause of cancer death in Australia, with only modest improvements in survival. This study aims to identify factors impacting on diagnosis and management of lung cancer with particular reference to Australian primary care. Methods: A sequential mixed method modified approach employing interview and a twophased survey technique. Following telephonic interviews with 31 health professionals (individuals representing general practitioners, specialized physicians, nurses and allied health practitioners), interview data was analysed using qualitative thematic analysis, and surveys using descriptive statistics. Emergent themes were organised under patient, provider and system factors. Interviews ceased upon saturation of data. Results: Multiple patient, provider and systems issues were seen to contribute to adverse health outcomes. There is a strong relationship between smoking and outcomes, and factors related to higher smoking rates such as a lower socioeconomic status. For smokers, guilt and/or denial was considered a reason for delay in the decision to seek medical care for cough or shortness of breath. Aboriginal people under-report morbidity related to smoking and chronic obstructive pulmonary disease; other patients fail to recognise the significance of their symptoms. Discussion: Despite the poor prognosis of lung cancer diagnosis, increased awareness of presentation and treatment options can address disparities in health outcomes.
\end{abstract}

Keywords: Lung Cancer; Primary Care; General Practice; Survival Diagnosis

\section{Introduction}

Lung cancer is the leading cause of death due to cancer in Australia. In 2005 there were 4711 deaths as a result of lung cancer [1]. It is currently the second leading cause of all deaths in men, and although incidence and mortality rates of lung cancer are declining in men, there are increasing rates in women [1]. Also of concern are the higher rates of lung cancer present in Indigenous Australians and those from socioeconomically deprived groups.

Except for short-term survival after a diagnosis of small cell lung cancer, the prognosis for patients with lung cancer has not greatly improved over recent decades [2]. Between 1998 and 2004 only 12\% of people with lung cancer survived five years [3,4]. As the population ages it is likely that the incidence of lung cancer will rise among older people [5]. In response to the increasing disease burden, there have been modest improvements in diagnosis, management and models of care delivery for lung cancer [6-8]. Due to the absence of screening methods, most patients with symptoms will consult a general practitioner or other primary care health professional before they are referred for specialist advice. The role of primary care professionals is essential at all levels of cancer care-from prevention and diagnosis through to palliation [9]. The ongoing relationship between patients and primary health care professionals provides the opportunity to assist patients and the families of those with lung cancer, and achieve the best possible outcomes.

The objective of this study was to identify factors that impact on the diagnosis and management of lung cancer with particular reference to Australian primary care.

\section{Methods}

This study used a mixed method approach. The benefit of using such an approach is that it allows for a broader, more inclusive approach accommodating a diverse range of opinions and addresses the limitations of traditional ap- 
proaches [10]. The first part of the study included interviews with expert clinicians $(n=31)$. This was followed by two national, online surveys of health professionals.

For the interviews, members of the research team identified expert participants $(n=31)$ on the basis of expertise, availability and clinical leadership roles. The participants included respiratory physicians $(n=6)$, medical oncologists $(n=4)$, palliative care specialists $(n=4)$, general practitioners $(n=4)$, individuals working within cancer policy implementation $(\mathrm{n}=3)$, Aboriginal health care workers $(n=3)$, public health physicians $(n=2)$, care coordinators $(n=2)$, a radiologist, a surgeon and a dietician. Length of consultations varied from 10 minutes to 45 minutes. Detailed notes taken at each consultation were analyzed using qualitative thematic analysis [11, 12]. Interviews ceased when there was saturation of data.

Participants in the two national, online surveys (Survey 1 and 2), were recruited by snowball sampling to ensure both broad coverage of health professionals, and national coverage using professional networks and a commercial distribution network. The survey was administered using a secure online platform. A targeted sampling method achieved representation from every state and territory, and representation across both the public and private health sectors. Item generation for the surveys was undertaken by a comprehensive literature review of published and grey literature, whilst questions were designed to elicit both barriers and facilitators to cancer care in Australia, and respondent's level of agreement to statements. The majority of questions were framed in a Likert score framework, with higher scores identifying a higher level of agreement.

\section{Results}

Survey 1 received 154 responses with a completion rate of $74.7 \%$ (137 respondents were included in the analysis). Not all respondents completed all questions, and correction for missing data was not undertaken as part of the analysis. Survey 2 elicited strategies to improve lung cancer care; 150 responses were received, with a completion rate of $73.3 \%$. The thematic analysis of interviews are presented as either patient, provider or system factors, as these have been identified as integral to driving health care reform [12]. Findings from the consultation process are summarized below under patient, provider and systems factors. In this study, we defined patient factors as those that influence patients' healthseeking behaviors and treatment adherence. Provider factors influence the capacity of health professionals to provide lung cancer services. System factors impact on the ability to provide and coordinate lung cancer services.

\subsection{Patient Factors}

Many of the contributing factors to variations in man- agement and outcomes in lung cancer are a function of patient circumstances and/or understanding. Socioeconomic factors exert a stronger influence in lung cancer than in most diseases due to smoking rates being higher in lower socioeconomic groups and among Aboriginal Australians. The issue of guilt arose throughout the consultations-for those who have smoked or who currently smoke, guilt can delay the decision to seek medical care, whilst shame resulting from failure to stop smoking is an important reason why individuals do not act on symptoms such as cough or shortness of breath.

Three experts considered that the Aboriginal population under-reported their morbidity related to smoking and chronic obstructive pulmonary disease (COPD). One Aboriginal health worker commented that "[There is] still a lot of racism in the system. It puts people off getting treatment. People feel shamed". In addition, the expert participants suggested that lung cancer is not always seen as a treatable health issue within the Aboriginal community and amongst primary healthcare professionals. One general practitioner from an Aboriginal medical service commented that "Patients present too late, and sometimes they are already expecting the answer because they see it as smoking related".

The high symptom burden associated with these, and also with functional decline and physiological changes in ageing, means that patients may fail to recognize the significance of their symptoms. Communication issues were also identified as patient factors contributing to variations in management. One dietician surveyed felt there existed a "need for more guidelines and marketing to patients and families".

In identifying patient factors impacting on the role of primary care in lung cancer diagnosis and management, the respondents emphasized:

- Targeting high risk groups to increase awareness of lung cancer in the absence of a valid and reliable screening test for lung cancer.

- Developing and testing models of intervention that empower consumers at high risk to monitor their health status for changes in symptoms.

- Informing professionals of the need to approach the discussion of smoking in clinical consultations as a strategy to decrease stigma associated with lung cancer and smoking.

- Providing access to care coordinators or lung cancer nurses to assist the patient in navigating the health care system.

\subsection{Provider Factors}

The small number of individuals with lung cancer presenting to an individual practitioner in primary care means that health professionals can be less attuned to the 
likelihood of lung cancer. Several GPs in this study stated they did not see lung cancer in their practice, and one acknowledged that "...I guess I am less switched onto it". This issue was also raised by a medical oncologist who commented that "physicians [are] not switched on to lung cancer". Similarly, a GP working in an Aboriginal medical service believed there should be an increased emphasis on considering the possibility of lung cancer in chronic care programs and adult health checks. Co-morbidity is highly influential as a provider factor. Symptoms such as cough and dyspnea are more likely to be attributed to COPD, CHD and CHF than lung cancer.

The consultations also elucidated provider factors including perceptions of nihilism and reluctance to refer to specialist providers. Experts stated that some health professionals may not be aware of the benefits of lung cancer treatment; as a consequence, they may be reluctant to refer patients for specialist treatment. One medical oncologist commented that: "Amongst some clinicians there is a perception of nihilism regarding treatment decisions".

The majority of respondents identified the need for improved diagnostic and technical solutions to improve care. Expert participants stated that although guidelines existed, there was less emphasis on risk assessment facilitating an early diagnosis. When asked to identify where clinical guidance materials would have the greatest impact, all areas identified received strong support, particularly during investigations leading to early diagnosis, guidelines for the diagnosis and staging, and outcomes for measuring the quality of lung cancer care.

\subsection{System Factors}

Respondents hinted at difficulties with access to timely diagnostics and treatment. Expert participants contend that a delay of up to four months in receiving treatment could occur, particularly in remote locations, although the reasons for reported delays were not confined to regional and remote centres. These delays are of concern in a condition with such a poor prognosis. One medical oncologist commented, "By the time the patient gets to the GP, the chest X-Ray gets reported, the patient goes back to the GP then goes to the respiratory physician who orders more tests, waits for the PET scan, then waits for a review appointment and then has a biopsy scheduled, weeks and sometimes months march on". Another commented "I have one patient who had three admissions to the emergency department with shortness of breath. Abnormal findings were noted on the chest X-Ray, but they still didn't get to the right specialist—and this is in a capital city".

A limited role for general practitioners in the treatment of patients with lung cancer was perceived. The role of primary care generally was seen as mainly focused on the coordination of care early in the patient trajectory, particularly with regard to ensuring that patients were being managed in a team approach. Systems and personnel that promote coordination, particularly lung cancer nurses and care coordinators, were endorsed as helpful in ensuring timely treatment. One medical oncologist commented that "multiple providers, multiple investigations can lead to delays in diagnosis". One radiologist maintained there is a "need for systematic followup of abnormal chest $x$-rays". This statement implies that systems for follow up are not in place.

\subsection{Strategies to Improve Outcomes}

A number of strategies to improve lung cancer care were identified by participants reflecting the three themespatient, provider and system. These are provided in order of frequency of responses in Table 1.

\section{Discussion}

In Australia, major obstacles to achieving improvements in outcomes for lung cancer remain, whilst primary care professionals are faced with multiple challenges. As noted during consultations, patient trajectory prior to entry into the health care system is reported to be highly variable. Experts in this study, however, suggested that once a correct diagnosis is made and lung specialists are engaged, there is less variability in management. Notwithstanding these observations, the respondents implied that there are unacceptable variations in lung cancer outcomes in Australia. Overall, as is noted by others, late presentation is the leading patient factor contributing to adverse health outcomes [13-15].

Table 1. Strategies to decrease variation in lung cancer outcomes.

\begin{tabular}{lc}
\hline Strategy & $\begin{array}{c}\text { Frequency of } \\
\text { response }(n)\end{array}$ \\
\hline $\begin{array}{l}\text { Educational initiatives for general practitioners } \\
\text { with strategies to increase the awareness and } \\
\text { recognition of symptoms }\end{array}$ & 72 \\
$\begin{array}{l}\text { Referral and access to multidisciplinary teams } \\
\text { (MDTs) to assist in diagnosis, staging and treatment }\end{array}$ & 65 \\
$\begin{array}{l}\text { Evidence based guidance to promote timely } \\
\text { investigation and diagnosis of lung cancer }\end{array}$ & 52 \\
$\begin{array}{l}\text { Strategies to increase public awareness of lung } \\
\text { cancer, diagnostic strategies and available therapies }\end{array}$ & 51 \\
$\begin{array}{l}\text { Recommendations to assist clinicians in monitoring } \\
\text { patients with ongoing symptoms when investigations } \\
\text { do not initially confirm lung cancer }\end{array}$ & 37 \\
\hline
\end{tabular}




\subsection{Patient Factors}

Consumer issues in lung cancer care are complex and are linked to physical, social, psychological and sociodemographic characteristics $[16,17]$. As reflected in interviews, the literature also emphasizes that presentation for treatment of lung cancer may be influenced by guilt associated with smoking, and this may be reflected in variations in lung cancer management, particularly in Indigenous Australians [15-20]. Notably, lung cancer incidence is significantly higher in the Indigenous population than in the non-Indigenous population in the 50 - 64 year age groups in the Northern Territory, Western Australia and South Australia [21]. Across all three jurisdictions, Indigenous populations had higher rates of smoking than the non-Indigenous populations [22]. A number of factors were identified as contributing to Indigenous Australians presenting late and, as a result, having a poor prognosis, with a very small proportion receiving treatment. This is consistent with literature recommending that, given the cultural diversity of Australia and high rates of smoking in some population groups, targeted culturally sensitive health information is required [21]. Pre-diagnosis barriers include knowledge of risk [13]. Factors of co-morbidity are also highly influential as reasons for late presentation by patients. Lung cancer is more common in older people, many of whom have multiple comorbid conditions $[23,24]$. Cough and dyspnea are symptoms commonly associated with smoking-related conditions such as COPD, coronary heart disease (CHD) and chronic heart failure (CHF) which may lead to dismissal of persistent symptoms.

\subsection{Provider Factors}

There are limited Australian data addressing specific provider factors impacting on diagnosis, referral and treatment-including thresholds for referral, follow-up after normal chest x-ray whether symptomatic or asymptomatic, knowledge of multidisciplinary teams (MDTs) and beliefs about the value of cancer specific treatment of lung cancer. However, reflecting the crucial role of the general practitioner (GP) as the gatekeeper to specialist services and early diagnosis [22], the role of the GP in monitoring individuals at high risk and facilitating the correct diagnosis was emphasized in this consultation of stakeholders. The reflections on nihilism are also consistent with the literature [25]. Compared to other cancers such as breast and bowel, there are minimal lung cancer education and training activities offered to primary care professionals [26]. Initiatives must therefore be devised that increase the awareness and knowledge of lung cancer amongst primary care professionals. GPs in particular need to be encouraged to explore the possibility of a lung cancer diagnosis.
With regard to access to treatment, the comments in the consultation are consistent with data indicating that in some instances, there is less than optimal access to treatment, and that reorganization of the health care system can afford benefits [27-29]. Consistent with this, a series of studies undertaken in NSW describes less than optimal use of radiotherapy, with the optimal utilization rate being between $45 \%$ and $68 \%$ at initial diagnosis [30].

\subsection{Systems Factors}

The major focus of recommendation for a systems approach was the emphasis on guidelines. These comments are reflected in the literature in which others have shown that developing clinical practice guidelines, augmented by implementation and monitoring strategies, can afford benefits [31,32]. An integrated mechanism of data management and monitoring must also occur at the systems level—this mechanism must be closely linked to clinical governance strategies.

Further guideline development for lung cancer care must occur with close consultation with not only health professionals, but non-governmental groups and consumers too. The data obtained indicated that, at a systems level, there must be continuing support and system-wide strategies to decrease smoking. Given the role smoking campaigns and media portrayals can play in increasing anxiety and stress [19], smoking campaigns must take into consideration the role of guilt and stigma and the consequences this can have on an individual.

Throughout the consultations, the importance of care and coordination associated with lung cancer management was underscored as being important. There is also increasing importance being placed on the role of multidisciplinary teams (MDTs) in lung cancer care [33,34]. Incentives must be provided and barriers reduced to allow primary care professionals to participate in MDTs. This will allow the increased coordination of care and monitoring of outcomes. For primary care professionals, challenges lie ahead in providing effective and beneficial healthcare across the private and public sectors.

\subsection{Strengths and Limitations}

Issues relating to sampling and measurement bias must be acknowledged, although all attempts were made to ensure a broad representation of opinion and geographic representation. Within the time and resources available, the team failed to engage a sufficiently large cohort of consumers with lung cancer to draw robust conclusions about their experience. Therefore, the views of consumers are not represented in this report. In spite of these limitations, this study provides a comprehensive description of factors impacting on lung cancer care in Australia from the provider perspective, and offers critical infor- 
mation to inform health services planning.

In conclusion, with the Australian health care system in a phase of dynamic health care reform, this study has identified a number of issues related to outcomes for lung cancer that may be encompassed within a reform agenda $[35,36]$. This study has identified patient, provider and system factors conspired to adversely impact on lung cancer outcomes. In order to meaningfully address the timely diagnosis and management of lung cancer, interventions need to address entrenched health disparities and more actively involve primary care professionals in identification and the coordination of care.

\section{Acknowledgements}

This paper is part of a larger project entitled Lung Cancer in Australia: Review of the Evidence, Consultation \& Research. This project received funding from the Australian Government through Cancer Australia.

\section{REFERENCES}

[1] Australian Institute of Health and Welfare, "Cancer in Australia: An Overview, 2008,” AIHW, Canberra, 2010.

[2] D. R. Youlden, S. M. Cramb and P. D. Baade, "Current Status of Lung Cancer in Queensland: 1982 to 2004,” Viertel Centre for Research in Cancer Control, The Cancer Council Queensland, Brisbane, 2007.

[3] Australian Institute of Health and Welfare, “Australia's Health 2010,” AIHW, Canberra, 2010.

[4] Australian Bureau of Statistics, "Causes of Death. Australia. 2008,” ABS, Canberra, 2010.

[5] W. D. Bolton, D. C. Rice, A. M. Correa, W. Hofstetter, R. Komaki, R. Mehran, K. Pisters, J. A. Roth, A. A. Vaporciyan and G. L. Walsh, "Influence of Age on Choice of Therapy and Surgical Outcomes in Patients with Nonsmall Cell Lung Cancer,” The American Surgeon, Vol. 75, No. 7, 2009, pp. 598-604.

[6] K. Yasufuku, T. Nakajima, K. Motoori, Y. Sekine, K. Shibuya, K. Hiroshima and T. Fujisawa, "Comparison of Endobronchial Ultrasound, Positron Emission Tomography, and CT for Lymph Node Staging of Lung Cancer," Chest, Vol. 130, No. 3, 2006, pp. 710-718. doi:10.1378/chest.130.3.710

[7] M. Coory, P. Gkolia, I. A. Yang, R. V. Bowman and K. M. Fong, "Systematic Review of Multidisciplinary Teams in the Management of Lung Cancer," Lung Cancer, Vol. 60 , No. 1, 2008, pp. 14-21. doi:10.1016/j.lungcan.2008.01.008

[8] I. A. Yang, K. M. Fong, P. V. Zimmerman, S. T. Holgate and J. W. Holloway, "Genetic Susceptibility to the Respiratory Effects of Air Pollution,” British Medical Journal, Vol. 85, No. 1006, 2009, pp. 428-436.

[9] B. R. McAvoy, J. M. Fletcher and M. Elwood, "Cancer Education and Training in Primary Health Care-A National Audit of Training Providers," Australian Family Physician, Vol. 36, No. 11, 2007, pp. 973-976.
[10] U. Kelle, "Combining Qualitative and Quantitative Methods in Research Practice: Purposes and Advantages," Qualitative Research in Psychology, Vol. 3, No. 4, 2006, pp. 293-311.

[11] A. M. Kilbourne, G. Switzer, K. Hyman, M. CrowleyMatoka and M. J. Fine, "Advancing Health Disparities Research within the Health Care System: A Conceptual Framework," American Journal of Public Health, Vol. 96, No. 12, 2006, pp. 2113-2121.

doi:10.2105/AJPH.2005.077628

[12] World Health Organisation, "Cancer Factsheet," WHO, Geneva, 2009.

[13] L. Hancock, R. S. Fisher, S. Redman, A. Reid and T. Tripodi, "Knowledge of Cancer Risk Reduction Practices in Rural Towns of New South Wales,” Australian and New Zealand Journal of Public Health, Vol. 20, No. 5, 1996, pp. 529-537. doi:10.1111/j.1467-842X.1996.tb01635.X

[14] M. Jiwa, G. Halkett, S. Aoun, H. Arnet, M. Smith, M. Pilkington and M. McMullen, "Factors Influencing the Speed of Cancer Diagnosis in Rural Western Australia: A General Practice Perspective,” BMC Family Practice, Vol. 8, No. 1, 2007, p. 27. doi:10.1186/1471-2296-8-27

[15] M. Jiwa, E. Maujean, K. Spilsbury and T. Threlfal, “The Trajectory of Lung Cancer Patients in Western Australia-A Data Linkage Study: Still a Grim Tale,” Lung Cancer, Vol. 70, No. 1, 2010, pp. 22-27. doi:10.1016/j.lungcan.2010.01.007

[16] S. Hall, C. D. J. Holman, T. Threlfall, H. Sheiner, M. Phillips, P. Katriss and S. Forbes, "Lung Cancer: An Exploration of Patient and General Practitioner Perspectives on the Realities of Care in Rural Western Australia," Australian Journal of Rural Health, Vol. 16, No. 6, 2008, pp. 355-362. doi:10.1111/j.1440-1584.2008.01016.X

[17] C. P. Gross, B. D. Smith, E. Wolf and M. Andersen, "Racial Disparities in Cancer Therapy: Did the Gap Narrow between 1992 and 2002?” Cancer, Vol. 112, No. 4, 2008, pp. 900-908. doi:10.1002/cncr.23228

[18] S. M. Smith, N. C. Campbell, U. MacLeod, A. J. Lee, A. Raja, S. Wyke, S. B. Ziebland, E. M. Duff, L. D. Ritchie and M. C. Nicolson, "Factors Contributing to the Time Taken to Consult with Symptoms of Lung Cancer: A Cross-Sectional Study,” Thorax, Vol. 64, No. 6, 2009, pp. 523-531. doi:10.1136/thx.2008.096560

[19] A. Chapple, S. Ziebland and A. McPherson, "Stigma, Shame, and Blame Experienced by Patients with Lung Cancer: Qualitative Study,” British Medical Journal, Vol. 328, No. 7454, 2004, p. 1470. doi:10.1136/bmj.38111.639734.7C

[20] S. Shahid, L. Finn, D. Bessarab and S. C. Thompson, "Understanding, Beliefs and Perspectives of Aboriginal People in Western Australia about Cancer and Its Impact on Access to Cancer Services," BMC Health Services Research, Vol. 9, No. 1, 2009, p. 132. doi:10.1186/1472-6963-9-132

[21] T. J. Threlfall, J. R. Thompson, Western Australian Cancer Registry and Department of Health Western Australia, "Cancer Incidence and Mortality in Western Australia, 2005,” Department of Health Western Australia, Perth, 
2007.

[22] National Health and Hospitals Reform Commission, “A Healthier Future for All Australians-Final Report June 2009,” NHHRC, ACT, 2009.

[23] D. B. Grose, G. Devereux, R. D. Jones, D. Sharma, C. Selby, K. Docherty, D. McIntosh, P. Downer, M. Nicolson and R. Milroy, "Variation in Practice, Comorbidity, and Treatment in Newly Diagnosed Lung Cancer Patients,” Journal of Clinical Oncology, 2010 ASCO Annual Meeting Proceedings, Post-Meeting Edition, Vol. 28, No. 15, 2010, Article ID: e12056.

[24] J. Ngeow, S. Leong, F. Gao, C. K. Toh, W. T. Lim, E. H. Tan and D. Poon, "Impact of Comorbidities on Clinical Outcomes in Non-Small Cell Lung Cancer Patients Who Are Elderly and/or Have Poor Performance Status," Critical Reviews in Oncology/Hematology, Vol. 76, No. 1, 2010, pp. 53-60. doi:10.1016/j.critrevonc.2009.10.005

[25] F. R. Khuri and J. Roman, "From Nihilism to Individualism: The Evolution of Lung Cancer Therapy,” American Journal of Respiratory and Critical Care Medicine, Vol. 177, No. 12, 2008, p. 1299. doi:10.1164/rccm.200802-339ED

[26] B. R. McAvoy, J. M. Fletcher and M. Elwood, "Cancer Education and Training in Primary Health Care-A National Audit of Training Providers," Australian Family Physician, Vol. 36, No. 11, 2007, pp. 973-976.

[27] K. M. Duvalko, M. Sherar and C. Sawka, "Creating a System for Performance Improvement in Cancer Care: Cancer Care Ontario’s Clinical Governance Framework,” Cancer Control: Journal of the Moffitt Cancer Center, Vol. 16, No. 4, 2009, pp. 293-302.

[28] M. Dahele, Y. Ung, J. Meharchand, H. Shulman, R. Zeldin, A. Behzadi, C. Simone, S. Cheng, C. Weigensberg and K. Sivjee, "Integrating Regional and Community Lung Cancer Services to Improve Patient Care,” Current Oncology, Vol. 14, No. 6, 2007, pp. 234-237. doi:10.3747/c0.2007.157

[29] S. S. Raab and D. M. Grzybicki, “Quality in Cancer Di- agnosis,” A Cancer Journal for Clinicians, Vol. 60, No. 3, 2010, pp. 139-165.

[30] S. K. Vinod, L. Simonella, D. Goldsbury, G. P. Delaney, B. Armstrong and D. L. O'Connell, "Underutilization of Radiotherapy for Lung Cancer in New South Wales, Australia," Cancer, Vol. 116, No. 3, 2010, pp. 686-694. doi:10.1002/cncr.24762

[31] M. P. Devbhandari, V. Joshi, P. Barber, P. Krysiak, R. Shah and M. T. Jones, "Active Treatment Rates for Lung Cancer in South Manchester: Are We Doing Enough?” Interactive CardioVascular and Thoracic Surgery, Vol. 11, No. 4, 2010, pp. 411-414. doi:10.1510/icvts.2009.227025

[32] I. M. Baig, P. Downer and R. Milroy, "Fighting Lung Cancer in the Developed World-A Model of Care in a UK Hospital,” Journal of Pakistan Medical Association, Vol. 61, No. 2, 2010, pp. 93-97.

[33] K. Bauman and D. Arenberg, "Multidisciplinary Evaluation of Patients with Suspected Lung Cancer,” Clinical Pulmonary Medicine, Vol. 17, No. 1, 2010, pp. 35-41. doi:10.1097/CPM.0b013e3181c849fe

[34] B. V. Naidu and P. B. Rajesh, "Developments in the Management of Patients with Lung Cancer in the United Kingdom Have Improved Quality of Care,” Proceedings of the American Thoracic Society, Vol. 5, No. 8, 2008, pp. 816-819. doi:10.1513/pats.200807-065TH

[35] H. K. Koh, S. C. Oppenheimer, S. B. Massin-Short, K. M. Emmons, A. C. Geller and K. Viswanath, "Translating Research Evidence into Practice to Reduce Health Disparities: A Social Determinants Approach," American Journal of Public Health, Vol. 100, No. S1, 2010, pp. S72-S80. doi:10.2105/AJPH.2009.167353

[36] E. Partridge and M. Fouad, "Community-Driven Approaches for Reducing Health Disparities in Cancer," Journal of the American Medical Association, Vol. 303, No. 11, 2010, pp. 1090-1091. doi:10.1001/jama.2010.282 